\title{
Clinical Pathways in Stroke Rehabilitation: Background, Scope, and Methods
}

\author{
Thomas Platz and Mayowa Owolabi
}

\section{Introduction}

Stroke remains the second leading cause of death and disability and one of the leading causes of depression and dementia globally (GBD 2015 Neurological Disorders Collaborator Group 2017; Owolabi et al. 2018). While stroke-related mortality standardized for age decreased over the last decades, the absolute number of new strokes (incidence), stroke-related deaths and stroke survivors living in our societies (prevalence) dramatically increased. From 1990 to 2010, the worldwide stroke prevalence increased by $15 \%$ from 435 on average to 502 per 100,000 people (Feigin et al. 2014) and then more recently by $21.8 \%$ from 2005 to 2015 for ischemic stroke globally and years lived with stroke-related disability by $22.0 \%$ (GBD 2015 Disease and Injury Incidence and Prevalence Collaborators 2016). This "dramatic" increase in strokerelated burden of disease and disability is foreseen to continue in societies around the globe due to ongoing epidemiologic transition and an ageing world population.

\footnotetext{
T. Platz $(\bowtie)$

Institute for Neurorehabilitation and Evidence-based Practice ("An-Institute", University of Greifswald), BDH-Klinik Greifswald, Greifswald, Germany

Neurorehabilitation Research Group, University Medical Centre Greifswald (UMG), Greifswald, Germany

Special Interest Group Clinical Pathways, World Federation Neurorehabilitation (WFNR), North Shields, UK

e-mail: T.Platz@bdh-klinik-greifswald.de

M. Owolabi

Department of Medicine, College of Medicine, University College Hospital, University of Ibadan, Ibadan, Nigeria

Blossom Specialist Medical Center, (First Center for Neurorehabilitation in East, West and Central Africa), Ibadan, Nigeria

Special Interest Group Clinical Pathways, World Federation for NeuroRehabilitation, North Shields, United Kingdom 
Fortunately, specialized inter-professional stroke care including rehabilitation can significantly reduce stroke-related disability and prevent the need to receive institutional care among stroke survivors (Stroke Unit Trialists' Collaboration 2013). In the Cochrane review, including 21 RCTs with a total of 39,994 participants, the risk to remain dependent (or die) after stroke could considerably be reduced compared to non-specialized care (OR $0.79,95 \%$ CI 0.68 to 0.90 ; $P=0.0007)$. Furthermore, there is ample meta-analytic Cochrane evidence that specific interventions developed for stroke rehabilitation reduce impairment and promote activities, examples are arm robot therapy (Mehrholz et al. 2018), treadmill training with partial body-weight support (Mehrholz et al. 2017a), or electromechanical gait training (Mehrholz et al. 2017b) to name a few. Without proper care, stroke survivors are at higher risk to remain dependent on carers, face heavy restrictions in their societal participation, and have to leave their homes and become nursing home residents.

Thus, there is an urgent need to promote, achieve, and sustain multidisciplinary stroke rehabilitation to tame the rapidly increasing burden of strokerelated disability worldwide. This is best performed by a multidisciplinary approach involving specialist doctors, nurses, and therapists from various disciplines with the best available external evidence being implemented in clinical practice.

But how should such teams know the most valid up-to-date evidence and thus take their decisions reliably in the best interest of their patient? How can the knowledge from clinical research be translated to everyday clinical practice so that stroke survivors regain independence with activities of daily living, participate in social life to the best possible degree, and maximize their quality of life?

Clinical pathways can be of great help for this purpose. They are documented tools that provide multidisciplinary teams with recommendations for appropriate care for a medical condition. When they are based on the best available up-to-date and valid evidence, they help to maximize achievement of treatment goals.

\section{Clinical Pathways}

Clinical pathways (CP) are structured multidisciplinary care plans for a certain condition (Campbell et al. 1998). They declare how in a standardized way multistep managed care of a clinical condition is meant to be performed at a given point of health care provision, i.e. locally. Based on three sentinel articles (Campbell et al. 1998; De Bleser et al. 2006; Vanhaecht et al. 2006), a Cochrane review on CPs identified five characteristic features of CPs: a CP is (1) a structured multidisciplinary plan of care, (2) promoting translation of evidence or guideline to local structures, (3) detailing steps in the course of treatment for a medical condition, (4) with time frames of criteria-based progression, and (5) aiming for standardization of care for a clinical condition (Rotter et al. 2010).

As such, they are suitable for the guidance and implementation of evidencebased interventions for stroke rehabilitation with the involvement of various health care disciplines, for the different clinical target domains (e.g. perception [somatosensory, visual], communication, swallowing, arm activities, mobility, cognition, 
and emotion) to be addressed during the time course after stroke, [i.e. the acute (up to 7 days), the subacute (first 6 months) and chronic phase], with implications for functional recovery and achievement of therapeutic goals (Bernhardt et al. 2017).

\section{The Evidence Gap}

The inherent challenge for the generation of evidence-based clinical pathways for such a complex issue as stroke rehabilitation is the very broad and rapidly expanding evidence base that needs to be taken into account. For any individual or health care centre, it is impossible to systematically search and critically appraise the relevant evidence even when one would restrict oneself to only the most relevant and valid research, i.e. randomized controlled trials (RCTs) and systematic reviews (SR) with meta-analytic data synthesis. Even national societies will hardly be able to cover all relevant evidence and perform an explicit critical appraisal when they generate their guidelines for stroke rehabilitation.

Anyone responsible for the generation of (local) stroke rehabilitation CPs will invariably face the following challenges:

While high-quality SR can provide valid and precise estimate of beneficial therapeutic effects, they do so only for a single type of intervention and one target syndrome. Hence, their coverage in stroke rehabilitation is rather restricted. Most frequently, they give no clue on how to decide between the various available therapeutic options when faced with a clinical question. In addition, even for their restricted scope they provide evidence, but refrain from giving explicit clinical practice recommendations. They are meant to provide an evidence synthesis, but do not incorporate the methodological structure to systematically deduce practice recommendations.

Guidelines, on the other hand, are more comprehensive in their coverage, yet have critical limits that may restrict their validity and applicability for CP development outside their primary societal context (Platz 2019). In some countries, recommendations for stroke rehabilitation were embedded in general stroke care guidelines or overall stroke rehabilitation guidelines, yet with a restricted evidence base; this may cause bias by evidence selection. In other countries, guidelines were limited to certain target domains within stroke rehabilitation (e.g. mobility) and thereby had a chance to be systematically evidence-based; here the restriction is their coverage in terms of clinical aspects in stroke rehabilitation. In addition, most of the available guidelines were developed in high-income countries and formulated for their specific national health care systems (Platz 2019). As such, they are not necessarily applicable in other nations, especially not in low- or middle-income countries with quite different health care context and practice settings.

Therefore, the development of valid up-to-date systematically evidence-based stroke rehabilitation $\mathrm{CP}$ is daunting. Nevertheless, two initiatives of the World Federation for NeuroRehabilitation (WFNR) might help to better achieve these goals in the future, one being a project on research for the provision of systematic evidence-to-decision knowledge covering both (a) a systematic best evidence synthesis based on systematic reviews and (b) a systematic multistep approach from the 
evidence to clinical practice recommendations (compare Platz et al. 2020 as an example), and the second being the evidence-based clinical practice recommendations for major topics in stroke rehabilitation provided in this book.

\section{$4 \quad$ International Provision of Practice Recommendations}

The evidence-based clinical practice recommendations, as presented in this book and authorized by the WFNR, systematically link best evidence synthesis with specific clinical practice recommendations for various key clinical problems faced in stroke rehabilitation. The recommendations do not go into organizational issues (i.e. how to organize the implementation) except for the final chapter. Any organizational recommendations related to individual clinical problems would likely only be valid for a restricted scope of health care systems. Implementation needs to take regional context and resources into account and is thus better addressed by local clinical pathway development, not international recommendations.

Other aspects integrated in the development of the practice guidelines presented in this book are (a) the coverage of individual chapters by international experts in the respective field, mostly being members of the corresponding Special Interest Groups (SIG) of the WFNR, (b) a multi-professional group of authors, (c) coming from different health care settings around the globe, and (d) a structured review process for the recommendations involving the panel of all book authors, further WFNR experts (non-authors), and stroke survivor representatives.

The rest of this chapter will present the scope, content, and methodology used for the generation of these practice recommendations in greater detail.

\section{Scope, Content, and Methodology Used for the Generation of the Practice Recommendations}

\subsection{Scope of the Evidence-Based Clinical Practice Recommendations}

The International Classification of Functioning, Disability, and Health (ICF) (World Health Organization 2001) is based on the biopsychosocial approach used to integrate the biological, individual, and social dimensions of health. The ICF distinguishes three components: (1) body functions and structures; (2) activity and participation; and (3) environmental and personal factors. While the organic brain damage causes deficits of body structures (i.e. of the brain) and function (so-called "impairments" such as paresis), the resulting activity limitations (e.g. reduced mobility) translate into participation restrictions (i.e. handicaps) depending on multiple individual and environmental factors.

Each chapter of this book addresses the assessment and treatment of the specific functional consequences of stroke that are related to breathing, swallowing, 
consciousness, cognition, emotion, communication, visual perception, motor functions, and activities including arm activities and mobility as well as driving after stroke.

Individual stroke survivors might be affected by one or more functional deficits with high inter-individual variation of degree of functional deficit and remaining functional capacity. Furthermore, these deficits change over time due to spontaneous recovery and therapeutic effects. Therefore, stroke rehabilitation treatment needs to be highly individualized. Most chapters of this book focus on the evidence for treatment effects in a specific dimension of function and provide valuable guidance on treatment decisions. This is to be implemented in the context of an individualized comprehensive rehabilitation care plan.

It is crucial to understand the overall current situation of a stroke survivor, to assess any functional strengths and weaknesses as well as individual goals for rehabilitation. Individualized treatment decisions across functional domains are then taken on that basis. Practice recommendations as provided in this book are not meant to be of a recipe-book character. They are rather subjected to the overall individualized rehabilitation goals and plan and apply whenever the rehabilitation plan addresses the clinical problem covered.

Since stroke rehabilitation works best when team-based (Stroke Unit Trialists' Collaboration 2013) and stroke rehabilitation frequently involves different professions such as neuropsychologists, occupational therapists, physiotherapists, speech and language therapists, sport therapists, nurses, and physicians, an interdisciplinary team is formed whenever possible. Accordingly, goal setting and team approach with the ICF used as framework are important in stroke rehabilitation and are addressed in Chap. 3.

Stroke rehabilitation starts within acute stroke care and remains a life-long endeavor in many cases. It takes place in various health care settings from the intensive care unit, the acute stroke care, and stroke rehabilitation unit, to the outpatient clinic, community-based, and domiciliary settings. These issues are discussed in Chap. 14 on health care settings in neurorehabilitation.

\subsection{Target Users of the Practice Recommendations}

Target population of the clinical pathways for stroke rehabilitation are physicians treating stroke survivors, especially neurologists and physiatrists, physiotherapists, (neuro)psychologists, nurses, occupational therapists, and speech and language therapists among other health care professionals involved in stroke rehabilitation.

Stroke survivors, their related proxy carer, stroke service providers, and politicians might also benefit from the pathways for their interest and purposes. The language necessary to portray the evidence and recommendations specifically might however not permit an easy understanding for non-professionals, even though the intention was to promote understanding across a broad audience. 


\subsection{Stakeholder Involvement}

\subsubsection{Practice Recommendations Developer Group}

The practice recommendation development group includes individuals from the various relevant professional groups, i.e. occupational therapists, physicians, physiotherapists, psychologists, and speech and language therapists. In addition, members of the group come from different continents and regions with diverse socioeconomical backgrounds. By these facts, the broadest representation of stroke rehabilitation scenarios by profession, region, and socioeconomical background was sought to be achieved.

For each member of the guideline development group, the following information is provided (see Table 1):

- name,

- discipline/content expertise (e.g. neurologist, physiotherapist),

- institution (e.g. City hospital),

- geographical location (e.g. Nigeria),

- description of the member's role in the guideline development group,

- conflict of interest statement

\subsubsection{Integration of Views and Preferences of the Target Population}

We created an feedback panel with all book authors, two neurorehabilitation expert clinicians sharing international responsibility within the WFNR who were not authors of chapters and who come from different socioeconomic backgrounds (the U.S.A. and Mexico), and four representatives of stroke survivor support groups from Germany (Stiftung Deutsche Schlaganfall-Hilfe; www.schlaganfall-hilfe.de/) and Europe (Stroke Alliance for Europe, SAFE is a non-profit organization that represents a range of stroke patient groups from across Europe; www.safestroke. $\mathrm{eu} /$ ). They all were invited to provide feedback on individual chapters and their recommendations.

Feedback given on individual chapter's recommendations by the panel of all authors, the two independent neurorehabilitation experts, and the representatives of stroke survivor support groups through a structured chapter-by-chapter webpagebased process was used by chapter authors to revise their chapter before it was accepted for publication.

\subsection{Methods Used for Evidence Synthesis and Recommendation Development}

\subsubsection{General Remarks}

The methods for evidence synthesis described below apply to a truly systematic review with critical appraisal of the literature. Given the resource restraints of the author groups, this was not possible for most of the chapters; the aspects fulfilled are 


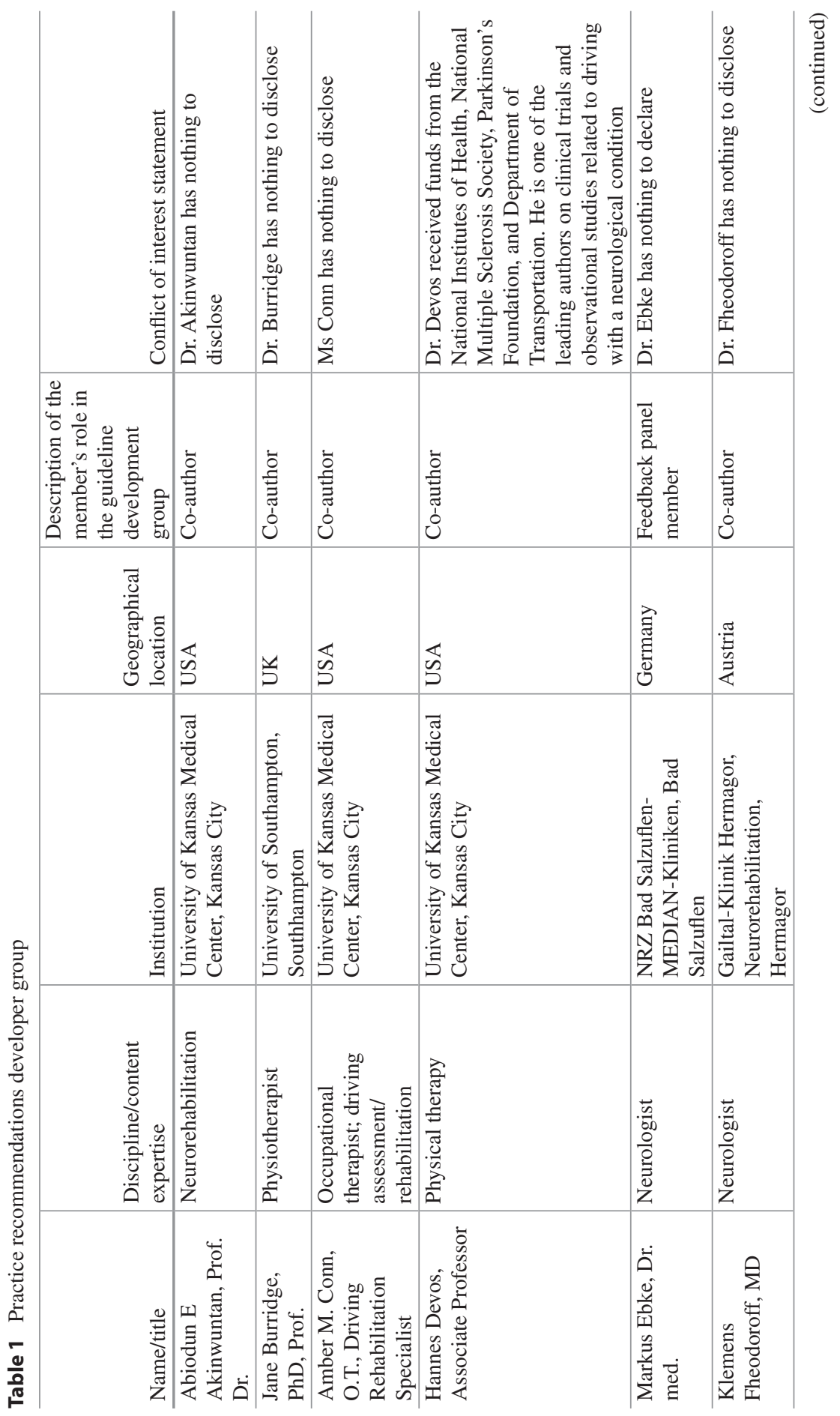




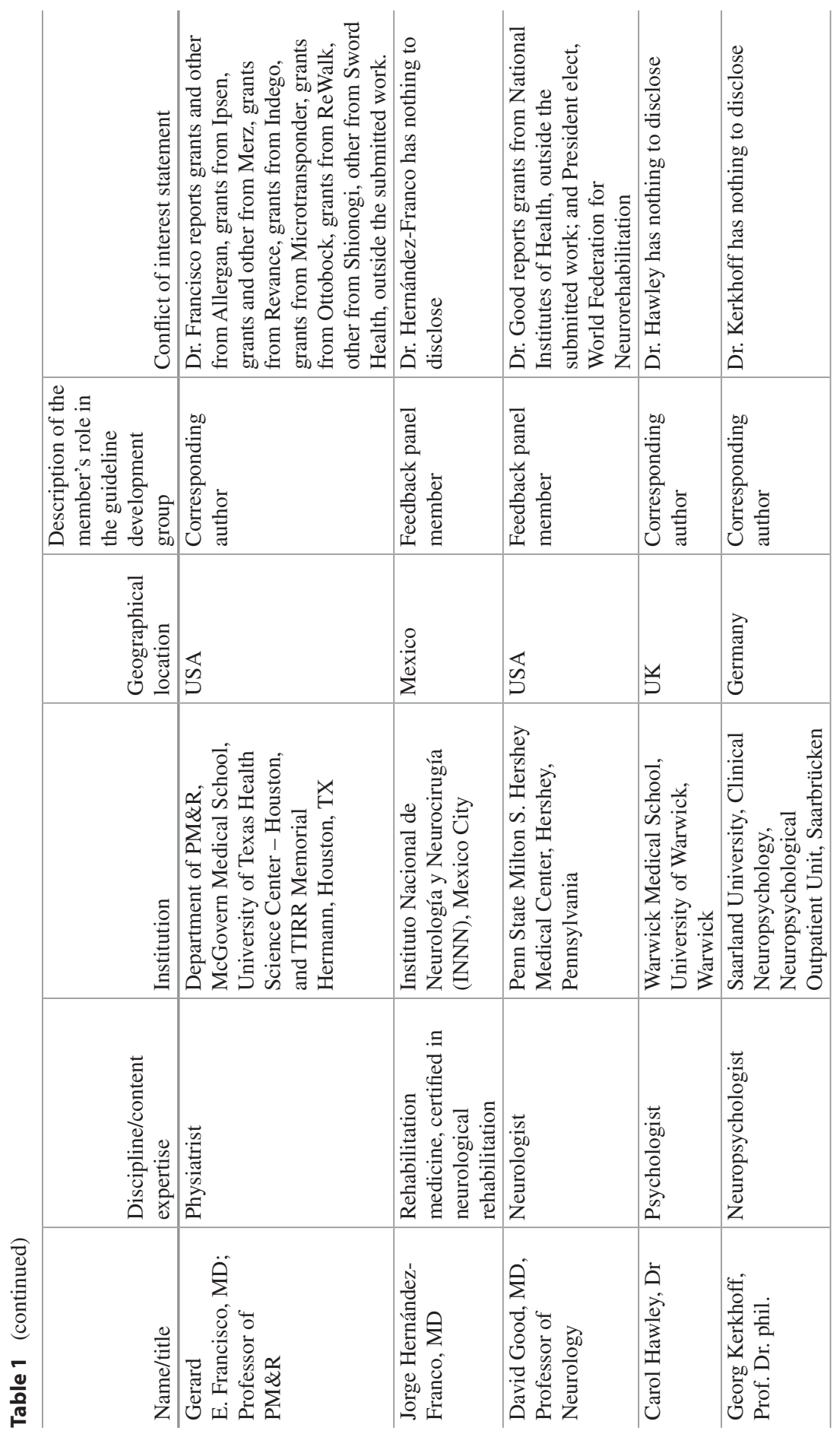




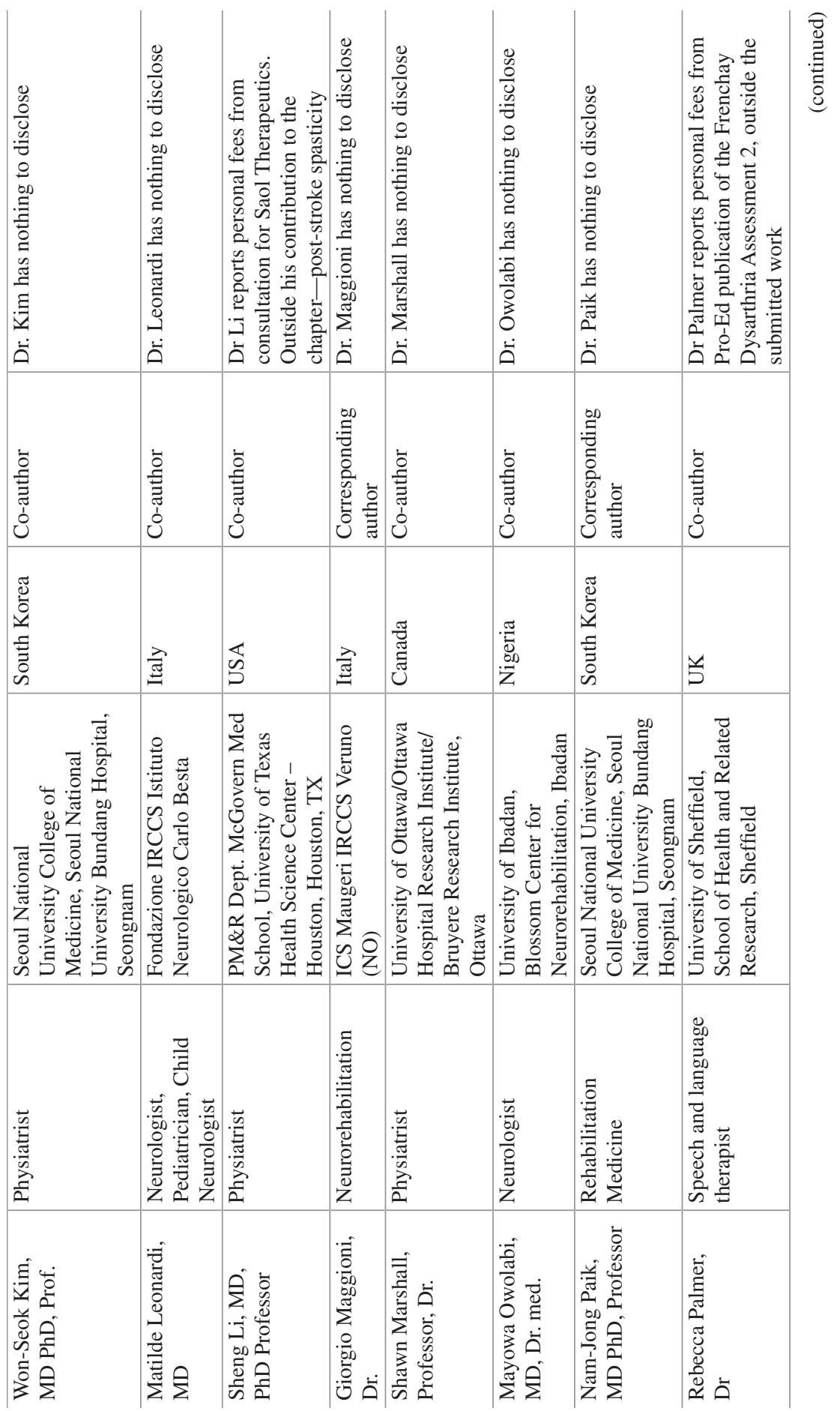




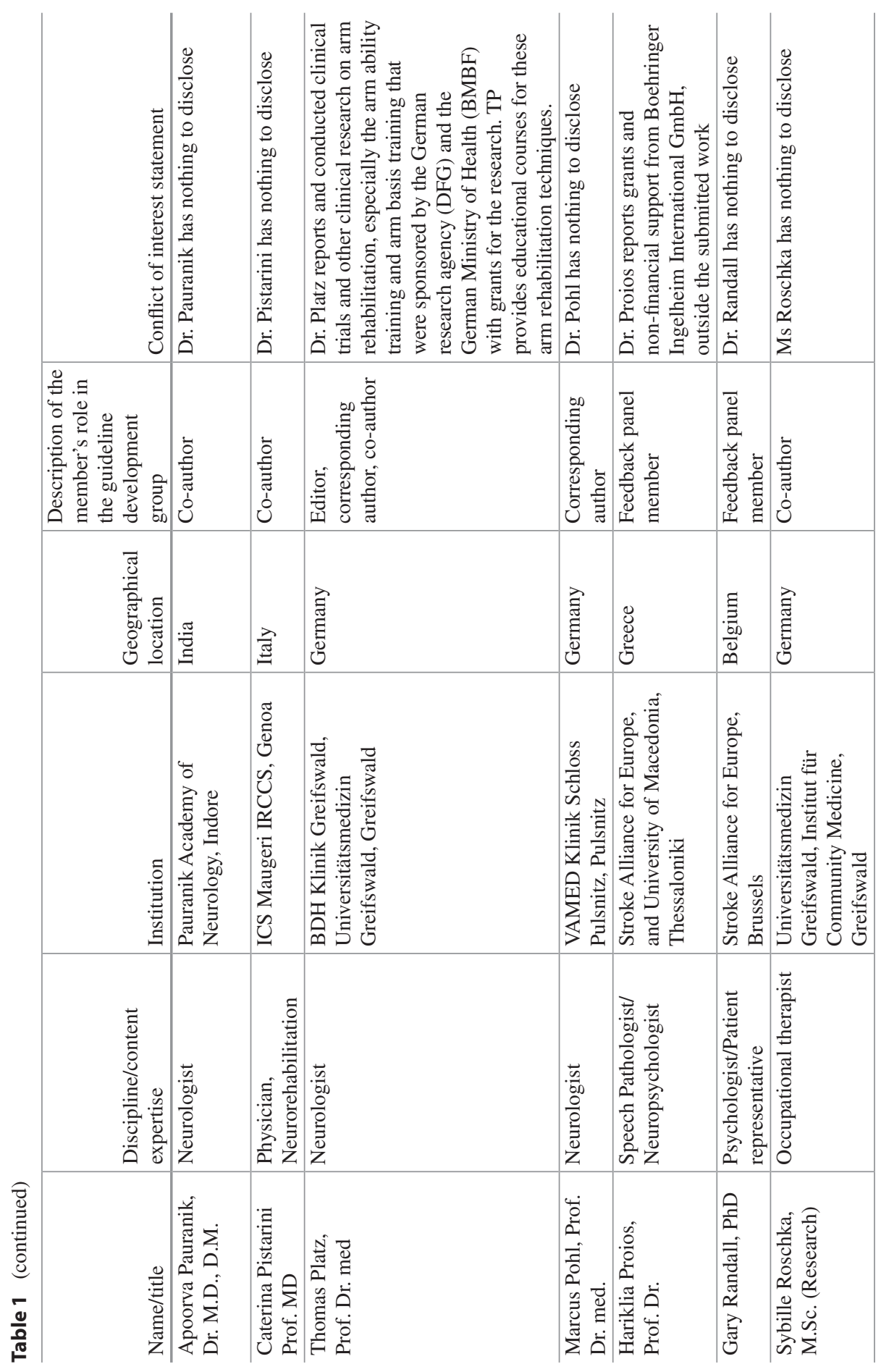




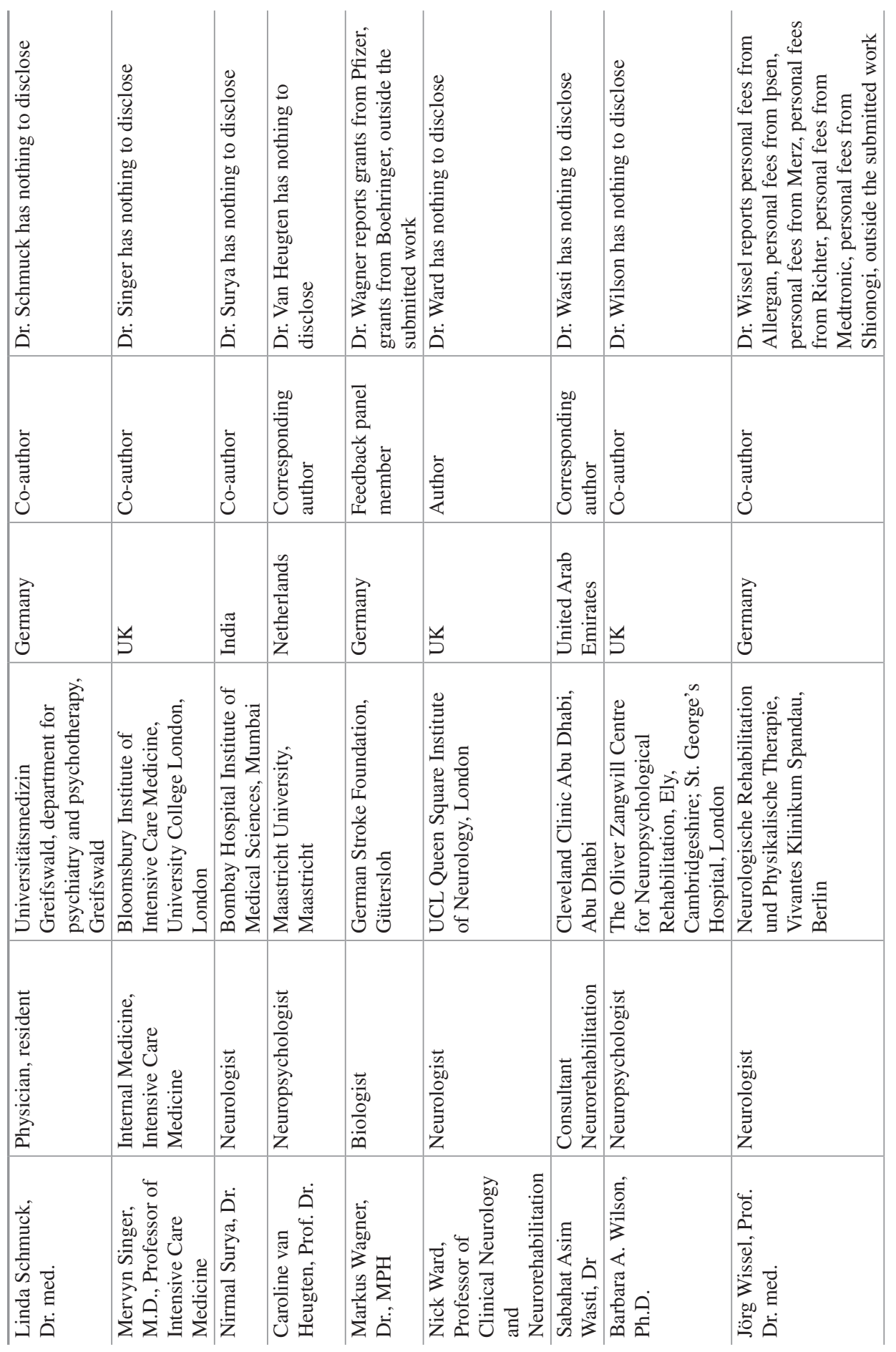


given in individual chapters. The methods presented here, nevertheless, describe the "gold standard".

In terms of the rules to assess the level of evidence of references, the quality of evidence, and the grading of recommendations, the methodology as described below was applied in all clinical chapters of this book.

\subsubsection{Systematic Search}

Details of the strategy used to search for evidence should be provided including search terms used, sources consulted, and dates of the literature covered. Sources included electronic databases (e.g. MEDLINE, EMBASE, CINAHL) and databases of systematic reviews (e.g. the Cochrane Library, DARE) and published conference proceedings. Other guidelines (e.g. the US National Guideline Clearinghouse, the German Guidelines Clearinghouse) could be used for comparison.

The information provided should include:

- named electronic database(s) or evidence source(s) where the search was performed (e.g. MEDLINE, EMBASE, PsycINFO, CINAHL),

- time periods searched (e.g. January 1, 2008 to April 30, 2018),

- search terms used (e.g. text words, indexing terms, subheadings),

- and may include the full search strategy (e.g. located in supplementary online material).

\subsubsection{Criteria and Methods for Evidence Selection and Data Extraction}

Criteria for including/excluding evidence should be provided. For example, some chapter authors decided to only include evidence from randomized clinical trials and to exclude articles not written in English. A description of the inclusion criteria included the target population (patient, public, etc.) characteristics, type of study design, intervention(s), comparison(s), outcome(s), language, and context, using an extended PICO schema (Lichtenstein et al. 2009).

Two independent assessors should perform evidence selection and data extraction. A consensus process should be in place to resolve any disagreement.

\subsubsection{Critical Appraisal, Level of Evidence, Evidence Synthesis, and Grading its Quality}

The following steps were taken from search for and critical appraisal of evidence to formulation of recommendations (Platz 2017, 2021) and are described in greater detail below.

I. For each source (original paper, systematic review, and meta-analysis)

1. evaluation of the methodology (internal validity, e.g. study design, risk of bias)

2. classification of evidence level of each source (1a to 5 according to the CEBM, for explanation see Table 1) (CEBM 2009) 
3. summarizing the results and their relevance for clinical practice based on individual sources.

II. For the collated data from all sources for a specific therapeutic intervention (original papers, systematic reviews, and meta-analyses)

4. assessment of the quality of evidence for the sources included, i.e. the resulting confidence in the estimate of the therapeutic effect strength (Schünemann et al. 2013) and.

5. formulating and grading of the derived recommendation (Schünemann et al. 2013; Platz 2017).

\section{Ad (I)}

Accordingly, for each reference and the body of literature for a given therapeutic intervention, the level of evidence was described (for details see Table 2).

Apart from data extraction and level of evidence classification, various aspects of trial validity should be critically appraised as presented in Table 3 for individual evaluation studies.

For systematic reviews, questions that are suggested to be addressed are given in Table 4. The criteria were adapted from AMSTAR 2 (A Measurement Tool to Assess Systematic Reviews) (Shea et al. 2017).

The characteristics of an individual study/systematic review together with the results of the critical appraisal, the main study results, and any clinical implications of that piece of information should be documented in an evidence table. There, the conclusion for individual references should specifically take into consideration the clinical relevance of the outcome measure(s), the magnitude and precision of the effect documented, the benefit-harm ratio, and the intervention's acceptability; methodological weaknesses/risk of bias; in case of meta-analyses subgroup analyses and heterogeneity; and finally, the relevance of findings for clinical practice.

Ad (II)

For any intervention-related recommendation, the quality of the evidence collated across all studies and systematic reviews included was assessed according to

Table 2 Level of Evidence Classification

\begin{tabular}{|c|c|c|c|c|c|}
\hline $1 \mathrm{a}$ & $1 b$ & $2 b$ & 3 & 4 & 5 \\
\hline $\begin{array}{l}\text { Systematic } \\
\text { review (with } \\
\text { homogeneity) } \\
\text { of RCTs }\end{array}$ & $\begin{array}{l}\text { Individual } \\
\text { RCT (with } \\
\text { narrow } \\
\text { confidence } \\
\text { interval) }\end{array}$ & $\begin{array}{l}\text { Individual } \\
\text { cohort study } \\
\text { or low- } \\
\text { quality RCT } \\
\text { (e.g. <80\% } \\
\text { follow-up) }\end{array}$ & $\begin{array}{l}\text { Individual } \\
\text { case- } \\
\text { control } \\
\text { study }\end{array}$ & $\begin{array}{l}\text { Case } \\
\text { series (and } \\
\text { poor- } \\
\text { quality } \\
\text { cohort and } \\
\text { case } \\
\text { control } \\
\text { studies) }\end{array}$ & $\begin{array}{l}\text { Expert opinion } \\
\text { without explicit } \\
\text { critical appraisal, } \\
\text { or based on } \\
\text { physiology, } \\
\text { bench research, } \\
\text { or "first } \\
\text { principles" }\end{array}$ \\
\hline
\end{tabular}

Levels of evidence for Therapy, Prevention, Aetiology and Harm 1a to 5 according to the "Oxford Center for Evidence-Based Medicine-Levels of Evidence", presented in table is the version from March 2009, retrieved from https://www.cebm.net/2009/06/oxford-centre-evidence-based-medicine-levels-evidence-march-2009/) (CEBM 2009). Alternatively, the classification from 2011 may be used (https://www.cebm.net/wp-content/uploads/2014/06/CEBM-Levels-of-Evidence-2.1.pdf) 
Table 3 Critical appraisal of individual evaluation studies

1. Clear definition of eligibility criteria.

2. Clear definition and adequate assessment of study outcomes.

3. Reporting of side effects and acceptability.

4. Adequate follow-up assessment (long-term effects).

5. Clear definition and description of experimental and control condition.

6. Were participants randomly allocated (selection bias)?

7. Allocation concealment (selection bias).

8. Comparability of experimental and control groups at baseline (selection bias).

9. Blinded staff and patients during intervention and comparable treatment of randomized groups aside from investigated effects (performance bias).

10. Blinded outcome assessment (detection bias).

11. No selective reporting (reporting bias).

12. (Almost) Complete outcome data (attrition bias).

13. Intention-to-treat analysis reported.

14. Do the results sufficiently support the conclusions reported?

Answers can be: yes (y), no (n), or not clear (nc).

Table 4 Critical appraisal of systematic reviews and meta-analyses

1. Were review methods established prior to the conduct of the review (written protocol)?

2. Were research questions clearly phrased, e.g. did selection criteria for the review include the components of PICO, and clinically meaningful?

3. Was the study design selection of included trials adequate for the research question?

4. Did the review authors use a comprehensive literature search strategy (data bases, key words, justify search restrictions [e.g. language])?

5. Were all processes (screening, selection, assessment risk of bias, data extraction) performed in duplicate?

6. Did the review authors describe the included studies in adequate detail (compare PICO)?

7. Did the review authors use a satisfactory technique for assessing the risk of bias (RoB) in individual studies that were included in the review?

8. If meta-analysis was performed, did the review authors use appropriate methods for statistical combination of results, and was it meaningful to combine the studies selected for meta-analyses?

9. Have all clinically relevant effects of the intervention(s) of interest (benefit, including long-term effects; harm; acceptability) been addressed?

10. Did the review authors assess the potential impact of RoB in individual studies and of publication bias on the results of the meta-analysis or other evidence synthesis and discuss the implications of the findings of their assessment on the estimates of therapeutic effects as reported?

11. Did the review authors provide a satisfactory explanation for and discussion of any heterogeneity observed in the results of the review?

12. Did the review authors report any potential sources of conflict of interest (CoI), including any funding they or the authors of included studies received for conducting the review or their studies? If a risk that CoI might have influenced the review's result is not unlikely, was its management described (for the review or the trials included) and adequate?

13. Do the results sufficiently support the conclusions drawn?

Answers can be:

yes (y), partially yes (py) [not all, but "essential features" yes], no (n), not clear (nc), or not applicable (na). 
Table 5 GRADE definition for quality of evidence

\begin{tabular}{|c|c|c|}
\hline $\begin{array}{l}\text { Quality of } \\
\text { evidence } \\
\text { category }\end{array}$ & Description & Examples \\
\hline High & $\begin{array}{l}\text { We are very confident that the true effect lies } \\
\text { close to that of the estimate of the effect }\end{array}$ & $\begin{array}{l}\text { Evidence from high-quality } \\
\text { RCTs or meta-analyses of } \\
\text { RCTs }\end{array}$ \\
\hline Moderate & $\begin{array}{l}\text { We are moderately confident in the effect } \\
\text { estimate: The true effect is likely to be close } \\
\text { to the estimate of the effect, but there is a } \\
\text { possibility that it is substantially different }\end{array}$ & $\begin{array}{l}\text { Evidence from RCTs or } \\
\text { meta-analyses of RCTs with } \\
\text { serious limitations } \\
\text { Evidence from observational } \\
\text { studies with special strengths }\end{array}$ \\
\hline Low & $\begin{array}{l}\text { Our confidence in the effect estimate is } \\
\text { limited: The true effect may be substantially } \\
\text { different from the estimate of the effect }\end{array}$ & $\begin{array}{l}\text { Evidence from observational } \\
\text { studies } \\
\text { Evidence from RCTs or } \\
\text { meta-analyses of RCTs with } \\
\text { multiple serious or very } \\
\text { serious limitations }\end{array}$ \\
\hline Very low & $\begin{array}{l}\text { We have very little confidence in the effect } \\
\text { estimate: The true effect is likely to be } \\
\text { substantially different from the estimate of } \\
\text { effect }\end{array}$ & $\begin{array}{l}\text { Evidence from observational } \\
\text { studies with serious limitations } \\
\text { Good clinical practice/expert } \\
\text { opinion }\end{array}$ \\
\hline
\end{tabular}

The quality of evidence according to the GRADE approach (Grading of Recommendations, Assessment, Development, and Evaluation) reflects the extent to which our confidence in an estimate of therapeutic effect is adequate to support a particular recommendation (left and middle column) (Schünemann et al. 2013). The right column provides examples how the categories were applied in the context of this book and its chapters.

the GRADE approach (Grading of Recommendations, Assessment, Development, and Evaluation) (Schünemann et al. 2013). The quality of evidence reflects the extent to which our confidence in an estimate of therapeutic effect is adequate to support a particular recommendation. The corresponding quality of evidence categories are presented in Table 5.

Randomized trials (and meta-analyses based on RCTs) without serious limitations provide high-quality evidence; observational studies without special strengths or serious limitations provide low-quality evidence. Limitations (risk of bias, inconsistency, indirectness, imprecision, and publication bias) or special strengths (e.g. large magnitude of effect or dose-response gradient) can, however, modify the quality of the evidence of both randomized trials and observational studies. For a more detailed explanation of risk of bias and other factors modifying the quality of the evidence, see the GRADE Handbook (Schünemann et al. 2013) and the Cochrane Handbook for Systematic Reviews of Interventions (Higgins et al. 2019; Schünemann et al. 2019).

\subsubsection{Synthesis of Evidence-Based Recommendations}

Recommendations were based on evidence for interventions and certain outcomes across studies and when available across systematic reviews. A recommendation reflects the extent to which the group developing the recommendation is confident 
that desirable effects of an intervention outweigh undesirable effects in case of a positive recommendation, or that undesirable effects of an intervention outweigh desirable effects in case of a negative recommendation.

GRADE specifies two categories of strength of recommendation, i.e. a weak or a strong recommendation in favour or against an intervention (Schünemann et al. 2013). For a strong recommendation, it is necessary to be certain about the various factors that influence the strength of recommendation and to have the information at hand that supports a clear balance towards either the desirable or the undesirable effects of an intervention. When the information is such that the desirable effects of an intervention probably outweigh the undesirable effects (or vice versa), but appreciable uncertainty exists, a weak recommendation for (or against) an intervention is warranted. In a first approach, high-quality evidence qualifies for a strong, moderatequality evidence for a weak recommendation.

In rehabilitation as in other medical fields, we frequently have some positive, yet low- or even very low-quality evidence favouring an intervention that strictly speaking is not yet sufficient to qualify for a weak recommendation. Nevertheless, this could still be the best available evidence and relevant for clinical guidance. Therefore, a third category of recommendation was introduced indicating a therapeutic “option” (Muche-Borowski et al. 2012; Platz 2017, 2021).

Furthermore, apart from the quality of evidence, other factors influence the grading of recommendations such as the clinical relevance of outcomes assessed, the value attributed by stroke survivors and the acceptability of a therapeutic option, the feasibility of its implementation, and the corresponding resource use. When such other factors contribute substantially to a recommendation's category, this was specifically indicated. Table 6 gives an overview of the recommendation categories used with the corresponding verbal descriptors for the text and the symbols used.

\subsubsection{Dissemination, Implementation, Monitoring, and Auditing}

This book is published under an open access schema. Thereby, it is accessible globally free of charge as electronic version and with a flat rate in print version. Being authorized by the WFNR, an umbrella organization for the national societies of neurological rehabilitation as well as for individuals working in countries without their own national society, a wide dissemination through national member societies

Table 6 Categories for recommendations

\begin{tabular}{l|l|l}
\hline $\begin{array}{l}\text { Recommendation } \\
\text { category }\end{array}$ & $\begin{array}{l}\text { Verbal description (as used in text) for positive/negative } \\
\text { recommendation }\end{array}$ & Symbol \\
\hline Strong & "Ought to"/“ought not to" & $\mathrm{A}+/ \mathrm{A}-$ \\
\hline Weak & "Should"/"should not" & $\mathrm{B}+/ \mathrm{B}-$ \\
\hline Option & "Can" & 0 \\
\hline
\end{tabular}

GRADE specifies two categories of strength of recommendation, i.e. a weak or a strong recommendation in favour or against an intervention, mainly for high- or moderate-quality evidence (Schünemann et al. 2013). A third category of recommendation was introduced indicating a therapeutic "option", mainly based on low- or very low-quality evidence (Muche-Borowski et al. 2012; Platz 2017, 2021) 
and key stakeholders worldwide is foreseen. While written in the "universal" language English, a language barrier for dissemination still needs to be taken into account. It is therefore intended to translate these practice recommendations into other languages to enhance their dissemination.

As stated above, contextualization, implementation, monitoring, and auditing of these practice recommendations relies on local initiatives. For instance, the recommendations can easily be used as building blocks for generating clinical pathways while taking the local health care settings into account. With their structured format they support both the formulation of local clinical pathways and can help to develop the regional health care architecture in a way that supports evidence-based stroke rehabilitation. Contextualized implementation cycles are suggested that engage all stakeholders such as providers (personnel, clinicians, healthcare workers), policymakers, patients, populace (communities), partners, and payers (Owolabi et al. 2016). This will motivate stakeholders, overcome the obstacle that guidelines developed in high-income countries are not easily applicable in low-and middle-income countries (Platz 2019), and create an enabling environment for the implementation of the evidence-based solutions presented. An illustration is given that addresses the interaction between WFNRauthorized practice recommendations and continuous quality improvement by use of contextualized clinical pathways, their communication, implementation, evaluation, and adjustment (compare Fig. 1).

WFNR: Evidence-based clinical practice recommendations for major topics in stroke rehabiliatiuon

Best available external evidence Concise, evidence-based, high level consensus recommendations

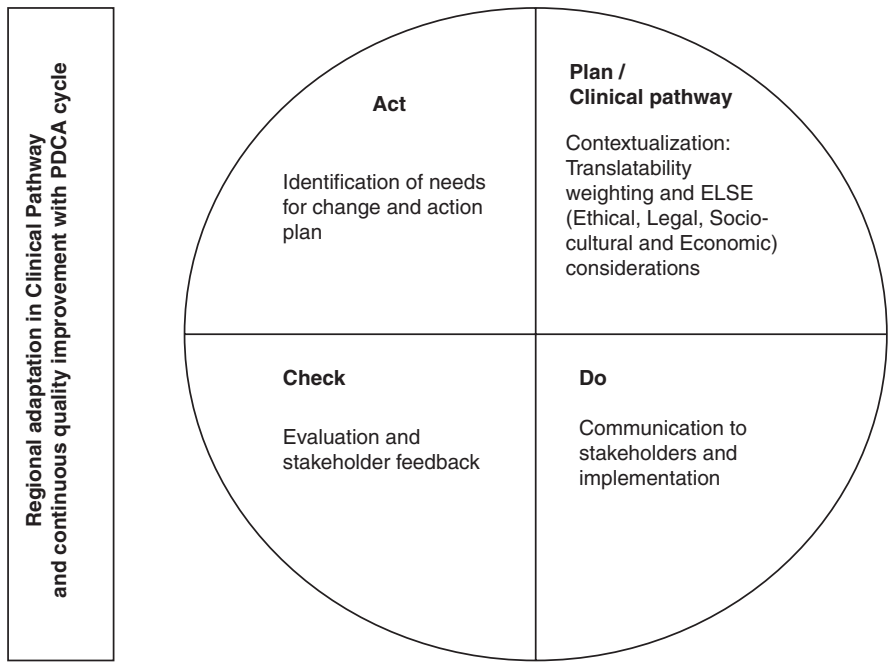

Fig. 1 Illustration of the interaction between WFNR-authorized practice recommendations and continuous quality improvement by use of contextualized clinical pathways, their communication, implementation, evaluation, and adjustment based on the plan-do-check-act (PDCA) cycle 


\subsubsection{Process of Updating the Clinical Practice Recommendations}

The practice recommendations are considered valid for 5 years from their time of publication and are intended to be updated thereafter.

\subsubsection{Funding of the Work}

The WFNR Research and Education Foundation sponsored the publication charges of this book under an open access schema. Thereby, its universal accessibility was supported. Other sources of funding that might apply for individual book chapters are noted in the respective acknowledgements. Funding did not involve commercial sources.

\section{Conclusions}

Stroke-related disability of any given stroke survivor is a consequence of a highly individual combination of various possible sensory, motor, cognitive impairments, emotional disorders, and associated activity limitations. Stroke rehabilitation aims to reduce disability and promote participation, while improving quality of life and sense of meaning and purpose in life (stroke recovery cycle) (Owolabi 2013). Related therapeutic goals are addressed by patient-tailored combinations of rehabilitation interventions that address specific stroke-related clinical problems. Evidence-based practice recommendations help to take clinical decisions related to these problems in a way that gives the best changes to promote functional recovery and to regain capacities to perform activities of daily living.

The evidence-based practice recommendations provided by the WFNR in this book are premised on a search for the best available valid up-to-date evidence, its critical appraisal, the collation of the evidence across trials, and systematic reviews in a clinical problem- and outcome-centred way. By knowing the evidence and judging its (un)certainty as well as other relevant aspects such as acceptability, feasibility, and resource implications, weak or strong recommendations (or therapeutic options) could be formulated both in favour or against an intervention of concern.

The degree of systematic search and critical appraisal varied across chapters in the book (as indicated in individual chapters) secondary to resources available for the work. The same methodology for classifying the level of evidence, grading the quality of evidence, and any recommendation given as outlined above was, however, used throughout this book.

Expert author groups provided both a best evidence synthesis and recommendations as draft versions for each clinical problem addressed. The recommendations were regarded as final and ready to be published after the contributions and feedback of the panel of all authors, further experts, and the representatives of stroke survivor support groups were incorporated.

These stroke rehabilitation practice recommendations are published under an open access schema (sponsored by the WFNR Research and Education Foundation) and distributed through the many national member societies for neurorehabilitation ensuring global dissemination. Together with knowledge about the regional health care settings, they can directly be used for the development of evidencebased clinical pathways for stroke rehabilitation locally. Their development was 
free of commercial funding. It is intended to provide an update 5 years after publication.

Acknowledgements This work was supported by the BDH Bundesverband Rehabilitation e.V. (charity for neuro-disabilities) by a non-restricted personal grant to TP. The sponsor had no role in the decision to publish or any content of the publication.

\section{References}

Bernhardt J, Hayward KS, Kwakkel G, Ward NS, Wolf SL, Borschmann K, Krakauer JW, Boyd LA, Carmichael ST, Corbett D, Cramer SC (2017) Agreed definitions and a shared vision for new standards in stroke recovery research: the stroke recovery and rehabilitation roundtable taskforce. Int J Stroke 12:444-450

Campbell H, Hotchkiss R, Bradshaw N, Porteous M (1998) Integrated care pathways. J Integr Care Pathw 316:133-137

CEBM (2009) "Oxford Center for Evidence-Based Medicine-Levels of Evidence", last version from March 2009. https://www.cebm.net/2009/06/oxford-centre-evidence-based-medicine-levelsevidence-march-2009/

De Bleser L, Depreitere R, De Waele K, Vanhaecht K, Vlayen J, Sermeus W (2006) Defining pathways. J Nurs Manag 14:553-563

Feigin VL, Forouzanfar MH, Krishnamurthi R, Mensah GA, Connor M, Bennett DA, Moran AE, Sacco RL, Anderson L, Truelsen T, O’Donnell M, Venketasubramanian N, Barker-Collo S, Lawes CM, Wang W, Shinohara Y, Witt E, Ezzati M, Naghavi M, Murray C, Global Burden of Diseases, Injuries, and Risk Factors Study 2010 (GBD 2010) and the GBD Stroke Experts Group (2014) Global and regional burden of stroke during 1990-2010: findings from the global burden of disease study 2010. Lancet 383:245-254

GBD 2015 Disease and Injury Incidence and Prevalence Collaborators (2016) Global, regional, and national incidence, prevalence, and years lived with disability for 310 diseases and injuries, 1990-2015: a systematic analysis for the global burden of disease study 2015. Lancet $388: 1545-1602$

GBD 2015 Neurological Disorders Collaborator Group (2017) Global, regional, and national burden of neurological disorders during 1990-2015: a systematic analysis for the global burden of disease study 2015. Lancet Neurol 16:877-897

Higgins JPT, Savovíc J, Page MJ, Elbers RG, Sterne JAC (2019) Chapter 8: Assessing risk of bias in a randomized trial. In: Higgins JPT, Thomas J, Chandler J, Cumpston M, Li T, Page MJ, Welch VA (eds) Cochrane handbook for systematic reviews of interventions. 2nd edition. Chichester (UK): John Wiley \& Sons, 205-228

Lichtenstein AH, Yetley EA, Lau J (2009) Application of systematic review methodology to the field of nutrition: nutritional research series, vol 1. Agency for Healthcare Research and Quality (US), Rockville, MD. 2009 Jan. (technical reviews, no. 17.1.) 2, methods. https://www.ncbi. nlm.nih.gov/books/NBK44075/

Mehrholz J, Thomas S, Elsner B (2017a) Treadmill training and body weight support for walking after stroke. Cochrane Database Syst Rev (8):CD002840

Mehrholz J, Thomas S, Werner C, Kugler J, Pohl M, Elsner B (2017b) Electromechanical-assisted training for walking after stroke. Cochrane Database Syst Rev (5):CD006185

Mehrholz J, Pohl M, Platz T, Kugler J, Elsner B (2018) Electromechanical and robot-assisted arm training for improving activities of daily living, arm function, and arm muscle strength after stroke. Cochrane Database Syst Rev (9):CD006876

Muche-Borowski C, Selbmann HK, Müller W et al. (2012) Das AWMF-Regelwerk Leitlinien 1. Aufl.; AWMF; http://www.awmf.org/leitlinien/awmf-regelwerk

Owolabi MO (2013) Consistent determinants of post-stroke health-related quality of life across diverse cultures: Berlin-Ibadan study. Acta Neurol Scand 128:311-320 
Owolabi MO, Miranda JJ, Yaria J, Ovbiagele B (2016) Controlling cardiovascular diseases in low and middle income countries by placing proof in pragmatism. BMJ Glob Health 1(3):e000105

Owolabi M, Johnson W, Khan T, Feigin V, for Operations Committee of the Lancet Neurology on Stroke (2018) Effectively combating stroke in low- and middle-income countries: placing proof in pragmatism - the lancet neurology commission. J Stroke Med 1:65-67

Platz T (2017) Practice guidelines in neurorehabilitation. Neurol Int Open 01(03):E148-E152. https://doi.org/10.1055/s-0043-103057

Platz T (2019) Evidence-based guidelines and clinical pathways in stroke rehabilitation-an international perspective. Front Neurol 10:200. https://doi.org/10.3389/fneur.2019.00200

Platz T, Vetter N, Falk M (2020) Treatment of neurovisual disorders post stroke - from systematic review evidence to clinical practice recommendations. PROSPERO CRD42020157933. Available from: https://www.crd.york.ac.uk/prospero/display_record.php?ID=CRD42020157933

Platz T (2021) Evidenzbasierte Leitlinienentwicklung der Deutschen Gesellschaft für Neurologie (DGN) und der Deutschen Gesellschaft für Neurorehabilitation (DGNR) - Methodik für die systematische Evidenzbasierung. Fortschritte der Neurologie - Psychiatrie, in press

Rotter T, Kinsman L, James EL, Machotta A, Gothe H, Willis J, Snow P, Kugler J (2010) Clinical pathways: effects on professional practice, patient outcomes, length of stay and hospital costs. Cochrane Database Syst Rev (3):CD006632

Schünemann H, Brożek J, Guyatt G, Oxman A (2013) GRADE handbook for grading quality of evidence and strength of recommendations. The GRADE Working Group. Updated October 2013. guidelinedevelopment.org/handbook

Schünemann HJ, Higgins JPT, Vist GE, Glasziou P, Akl E, Skoetz N, Guyatt GH (2019) Chapter 14: Completing 'Summary of findings' tables and grading the certainty of the evidence. In: Higgins JPT, Thomas J, Chandler J, Cumpston M, Li T, Page MJ, Welch VA (eds) Cochrane handbook for systematic reviews of interventions. 2nd edition. Chichester (UK): John Wiley \& Sons, $375-402$

Shea BJ, Reeves BC, Wells G, Thuku M, Hamel C, Moran J, Moher D, Tugwell P, Welch V, Kristjansson E, Henry DA (2017) AMSTAR 2: a critical appraisal tool for systematic reviews that include randomised or non-randomised studies of healthcare interventions, or both. BMJ 358:j4008

Stroke Unit Trialists' Collaboration (2013) Organised inpatient (stroke unit) care for stroke. Cochrane Database Syst Rev (9):CD000197

Vanhaecht K, De Witte K, Depreitere R, Sermeus W (2006) Clinical pathway audit tools: a systematic review. J Nurs Manag 14:529-537

World Health Organization (2001) International classification of functioning, disability and health. World Health Organization, Geneva

Open Access This chapter is licensed under the terms of the Creative Commons AttributionNonCommercial-NoDerivatives 4.0 International License (http://creativecommons.org/licenses/ by-nc-nd/4.0/), which permits any noncommercial use, sharing, distribution and reproduction in any medium or format, as long as you give appropriate credit to the original author(s) and the source, provide a link to the Creative Commons license and indicate if you modified the licensed material. You do not have permission under this license to share adapted material derived from this chapter or parts of it.

The images or other third party material in this chapter are included in the chapter's Creative Commons license, unless indicated otherwise in a credit line to the material. If material is not included in the chapter's Creative Commons license and your intended use is not permitted by statutory regulation or exceeds the permitted use, you will need to obtain permission directly from the copyright holder. 